\title{
OPTICAL SURVEYS OF THE MAGELLANIC BRIDGE, STREAM AND OUTER HALOS
}

\author{
M.J. IRWIN \\ Institute of Astronomy \\ Cambridge $\mathrm{CB} 3 \mathrm{OHA}$ \\ England
}

\begin{abstract}
The HI envelope of the Magellanic System extends well beyond the obvious visible regions of each of the Clouds. Modern optical surveys based on UK Schmidt telescope plates and fast measuring machines are now starting to reveal the complex optical counterparts of some of these HI features.
\end{abstract}

\section{Introduction}

The dynamical models of a tidally interacting, bound Galaxy-LMC-SMC system of Murai \& Fujimoto (1980) predict that there were several previous perigalactic encounters between the Magellanic Clouds and the Galaxy and that the SMC and LMC were within $3 \mathrm{kpc}$ of each other $\sim 2$ $\mathrm{x} 10^{8} \mathrm{yr}$ ago. As noted by Westerlund (1989), given the uncertainties of the model, the observational evidence of episodic bursts of star formation within the Clouds and the general properties of the HI distribution provide convincing corroborative proof. However, many of the expected optical counterparts to these dramatic events have proven surprisingly difficult to locate. This review summarises the current status of searches for the outlying optical manifestations of the interaction between the Clouds and the Galaxy.

\section{The morphology of the Outer Halos of the Magellanic Clouds}

Low resolution surface photometry of the Clouds (de Vaucouleurs 1955, de Vaucouleurs \& Freemen 1972) show the LMC extending over a roughly rectangular region $15^{\circ} \times 13^{\circ}$, whilst the SMC outer isophote is more elliptical and covers an area $7^{\circ} \times 4^{\circ}$. Surrounding the LMC are several regions of complex foreground nebulosity, the most striking of which is a $20^{\circ}$ arc running from $4^{h} 45,-73^{\circ}$ to $3^{h} 25,-57^{\circ}$. Originally thought to be an outer spiral arm of the LMC, this arc is now known to be galactic reflection nebulosity within $1 \mathrm{kpc}$ of the sun (McGee et al. 1986, Irwin et al. 1990a), illustrating the difficulties of studying the outer envelope of the Clouds by surface photometry alone.

The cluster system of the Clouds as derived from wide field survey plates (see Olszewski et al. 1988, Brück 1975 and references therein) is shown in Fig 1. Overall, the extent of the clusters is similar to that found from the low resolution photographs, though the LMC cluster system is more nearly elliptical, $15^{\circ} \times 11^{\circ}$, as noted by Lynga and Westerlund (1963). Lynga and Westerlund also pointed out that the southern "arm" of clusters coincided with features from de Vaucouleurs' (1955) deep photographs. Of the real LMC outliers, NGC1466, 1841, 2257 have velocities and distances consistent with LMC membership; Reticulum, E2, 121-SC03 are the same 


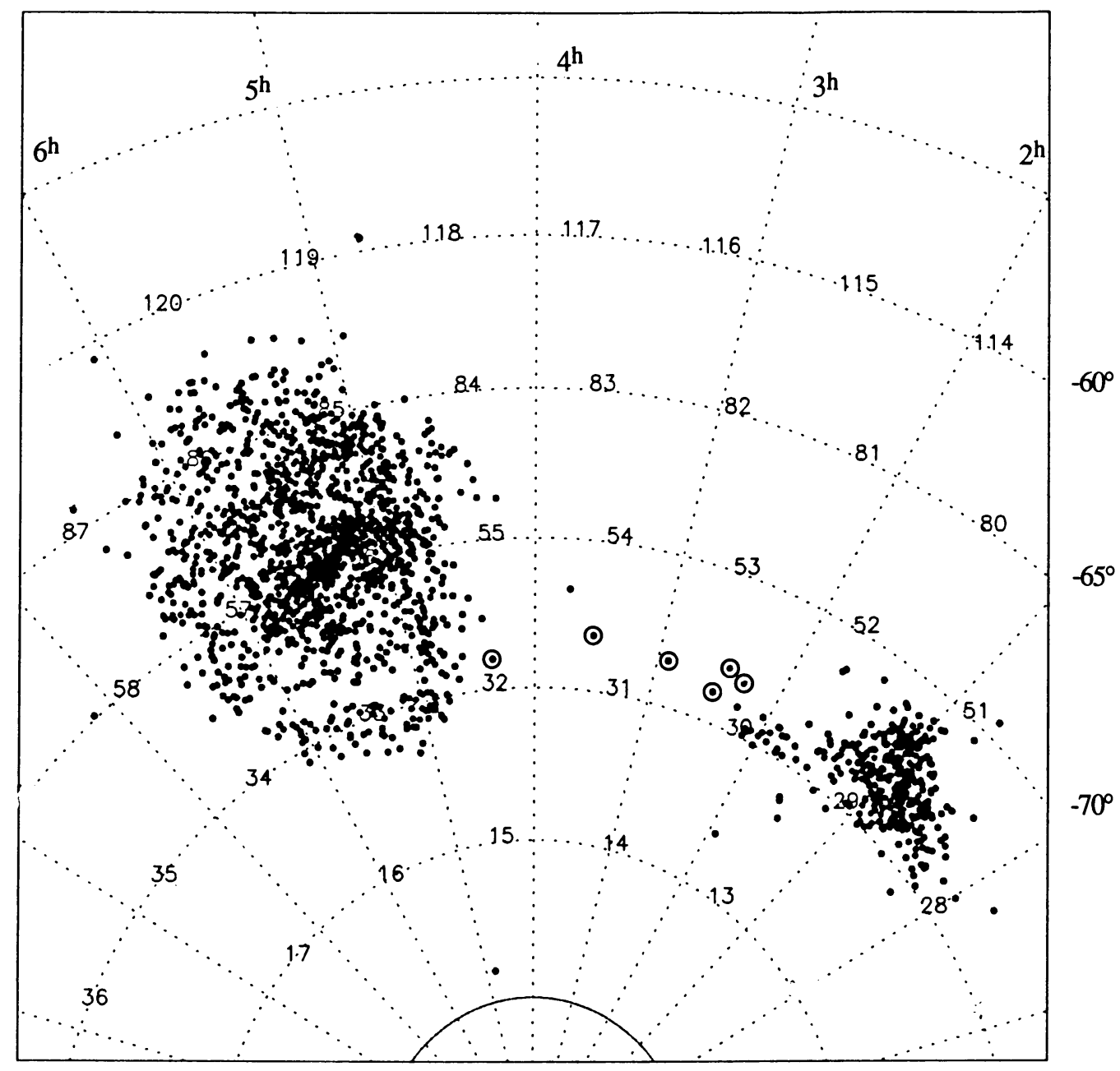

Figure 1. The distribution of Magellanic Cloud clusters is shown on a grid of UKST field numbers. The blue stellar associations found by Irwin et al. (1990) are shown with encircled symbols. 
distance as the LMC but as yet have no published velocities; OL-37 has not been studied (see Olszewski 1988 for nomenclature etc.). For the SMC little is known about most of the outlying clusters, other than $\mathrm{L} 1$ and NGC121 - both at distances compatible to the SMC. AM-3, a faint cluster discovered by Madore and Arp (1979), is a candidate for the westernmost cluster of the SMC. These remote clusters of both Clouds apparently lie much further out than the limits seen in either the HI distribution or the low resolution photographic survey, yet seem to be associated with the Clouds.

Previous machine-based measurements of UKST plates were mainly concerned with the SMC (e.g. Brück 1979, 1980) and revealed a halo population surrounding the SMC to a radius of more than $5^{\circ}$. Albers et al. (1987) detected shell-type features to the NE of the SMC which seemed to be connected with similar structure seen in the SMC wing. More recently, Hatzidimitriou and Hawkins (1989) have examined the three-dimensional structure of outlying stellar populations of the SMC using the width of the horizontal branch/red giant clump as an indicator of line-of-sight depth, and find significant variations of depth ranging from $10 \mathrm{kpc}$ in the $\mathrm{SW}$ to $23 \mathrm{kpc}$ in the NE. The extent of the SNC can be seen clearly in Fig 2 and is well described by a circle with diameter $\sim 14^{\circ}$, significantly larger than the nominal tidal radius of $\sim 4^{\circ}$. This mosaic of stellar isopleth (isodensity) maps to a limiting magnitude of $\mathrm{B}_{J} \sim 22$ was made from APM measures of UKST IIIaJ plates; all of the outlying SMC clusters lie within the boundaries of the SMC halo. Note particularly that the cluster AM-3 lies within the outer isophote of the SMC and is obviusly a good candidate for SMC membership. The extension of the SMC wing into the bridge region is also clearly visible as is the proximity of the LMC halo (see also Fig 3.). The disturbed isophotes to the $\mathrm{E}$ of the SMC near the wing, noted by Albers $e t$ al. are also readily apparent.

Similar APM measurements around the LMC are illustrated in Fig 3. Once again it is obvious that the outer halo of the LMC extends much further than previous surveys revealed, and actually envelopes all of the previously mentioned clusters, except perhaps NGC1841 where the isophotal structure becomes somewhat irregular. The region to the NE of the LMC is rather close to the Galactic plane; the outward distortion of the NE outer isophotes is caused by this. Shell-like irregularities of the isophotal contours are clearly visible to the SW at around $4^{h} 55,-75.5^{\circ}$ (UKST field 32). This is also in the direction where the outer isodensity structure is distorted and close to the easternmost blue SMC cluster found by Irwin et al. 1990b. Overall we can see that the extent of the LMC halo is an ellipse $\sim 23^{\circ} \times 17^{\circ}$, in good agreement with the ellipticity of the cluster system and once again somewhat larger than the nominal tidal radius of $\sim 8^{\circ}$. Freeman et al. (1983) showed that the LMC clusters form a flattened rotating "disk" system consistent with an inclination of the disk of $\sim 27^{\circ}$, with the $\mathrm{W}$ of the LMC pointing toward the SMC. The cluster data and the optical isophotes both imply an inclination angle closer to $\sim 45^{\circ}$ (see also Westerlund 1989). For all the outer fields surrounding the LMC there is no evidence of any significant broadening of the horizontal branch/red giant clump over and above that caused by photographic errors and the intrinsic stellar dispersion, in good agreement with a flattened rotating disk model.

\section{The Magellanic Bridge}

The survey of Hindman et al. (1963) revealed for the first time that a bridge of gas existed between the Magellanic Clouds. Later observations (e.g. Mathewson et al. 1979 and references therein) showed a complex HI structure with an underlying smooth velocity gradient across the whole bridge region strongly suggesting a bound system. Since in the majority of the Magellanic system there is a strong correlation between HI density and the presence of stellar condensations, this led to speculation that stellar associations may be present in the HI extension leading away from the optical wing of the SMC toward the LMC. 


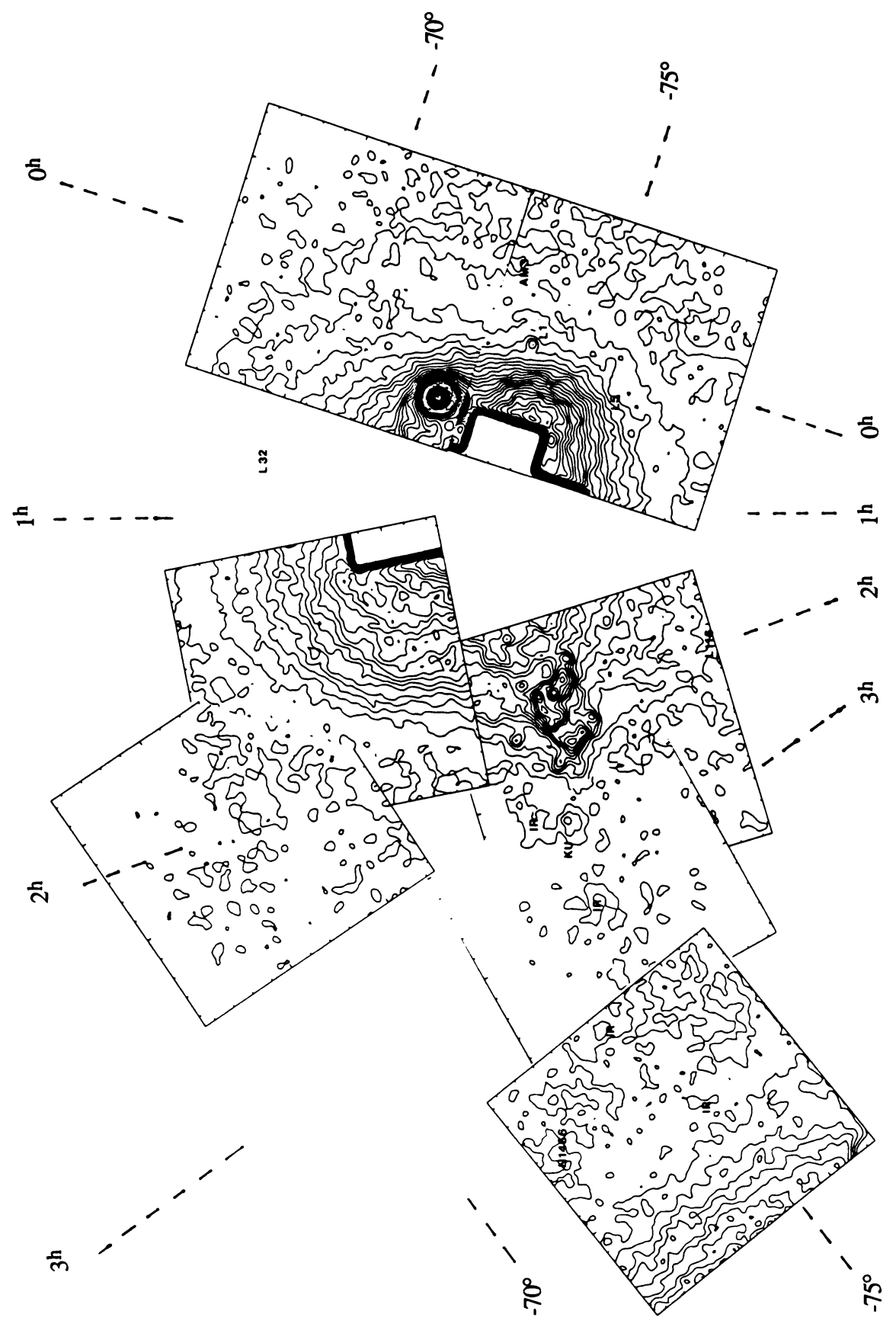

Figure 2. A mosaic of stellar isopleth maps of the SMC and bridge region. Contour levels start at $\sim 2.0$ images $\operatorname{arcmin}^{-2}$ and increase in steps of 0.5 images $\operatorname{arcmin}^{-2}$. Potential outlying clusters of the SMC are indicated. 


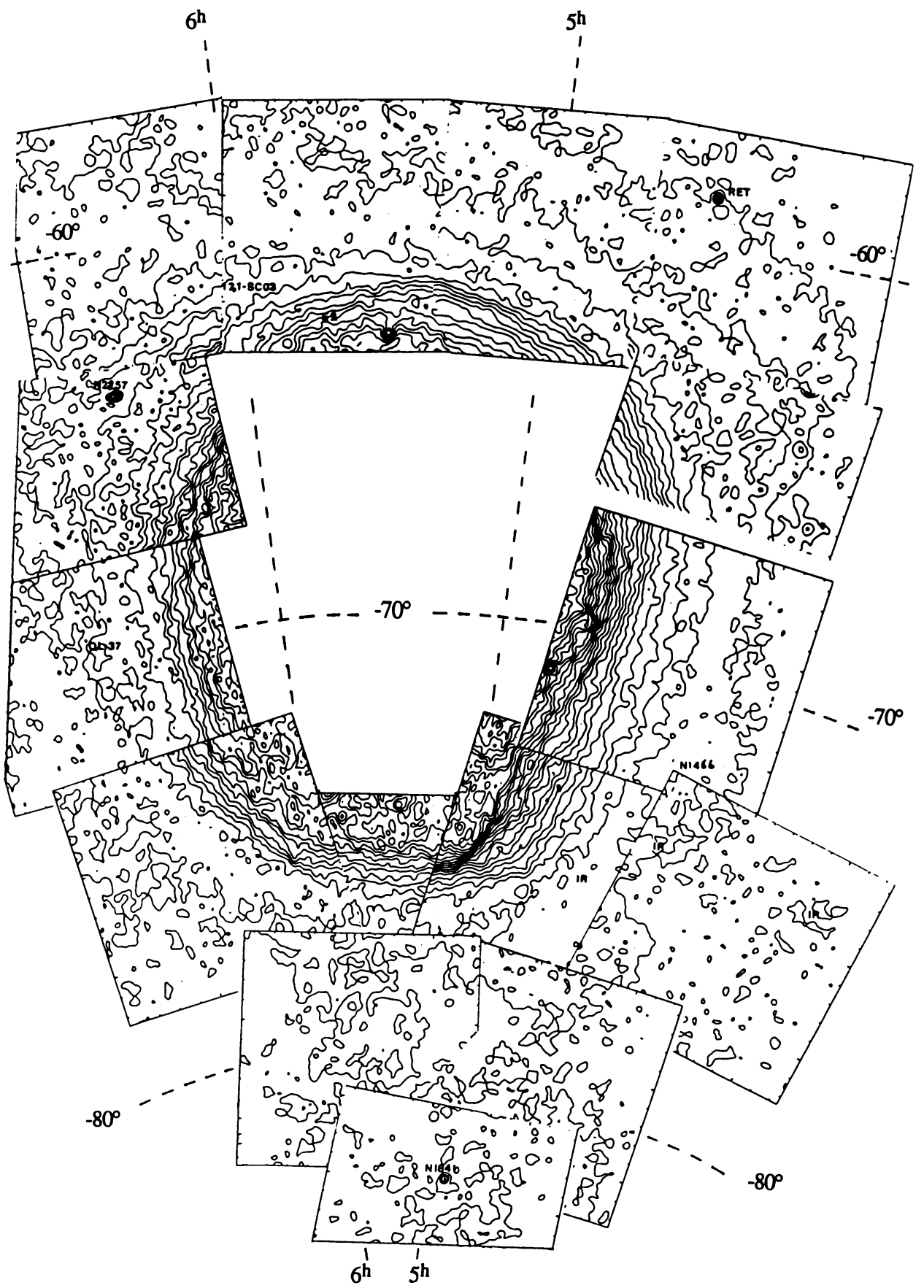

Figure 3. A mosaic of stellar isopleth maps of the LMC. Contour levels start at $\sim 2.0$ images $\operatorname{arcmin}^{-2}$ and increase in steps of 0.5 images $\operatorname{arcmin}^{-2}$. Note how all the outlying potential LMC clusters lie within the outermost contour level. By combining this diagram with Fig. 2 it is readily apparent that the halos of the Clouds essentially overlap. 
In the past this inter-Cloud region received little attention from optical astronomers. Sanduleak (1969) carried out an objective prism survey of the area but failed to find bright Cloud members $\left(\mathrm{m}_{p g}<14\right)$ in the inter-Cloud region. Wamsteker $(1981)$ published photoelectric photometry of $\sim 500$ stars $(\mathrm{V}<14.5)$ between the Clouds. However, nearly all the early-type stars observed were in the known SMC wing, which extends to $2^{h} 15,-74^{\circ}$; no Cloud members were found in the bridge region. The eastern end of the SMC wing at $1^{h} 50.0,-74^{\circ} 15$ was also found to coincide with a region of UV emission detected by a space-lab experiment (Courtes et al. 1984) and $\mathrm{H} \alpha$ emission was reported by Marcelin et al. (1985) and Meaburn (1986) in the same area, though a field further to the east at $2^{h} 10,-74^{\circ} 34$ gave a null result. Johnson et al. (1982), using deep wide-field $\mathrm{H} \alpha$ image tube photographs found a very diffuse region of $\mathrm{H} \alpha$ emission between the Clouds which appeared to be correlated with the HI distribution. However, previous work on star counts across the bridge region by Brück (1980) failed to reveal any link between the Clouds. In contrast, Kunkel (1980) using CTIO $4 \mathrm{~m}$ plates centred on $2^{h} 30,-74^{\circ}$ found a group of young stellar associations (age $\sim 10^{8}$ years) coincident with a dense HI cloud. Lying $8^{\circ}$ from the centre of the SMC, well outside any previously known optical component, this represented the first unambiguous optical detection of the HI bridge.

In a follow-up to Kunkel's discovery, Irwin et al. $(1985,1990$ a) used the APM facility to process three bairs ( $B_{J}$ and OR) of contiguous UKST plates centred on $2^{h} 00,-68^{\circ} ; 2^{h} 48,-74^{\circ}$ and $4^{h} 00,-73^{\circ}$, which covers the main body of the bridge region and the spur of HI to the NE of the SMC. Colour-magnitude diagrams obtained from the plate pairs clearly showed the presence of blue main-sequence stars between the wing of the SMC and the western halo of the LMC. The spatial distribution of the blue stars, $\mathrm{B}^{J-} \mathrm{R}<0$ shows that many of them are in aggregates and newly identified clusters, stretching from an area surrounding and including the SMC wing tip all the way across to the LMC halo. Spectroscopic observations confirmed that the stars are indeed OB stars at a distance of $\sim 50 \mathrm{kpc}$ with radial velocities consistent with Cloud membership, confirming that the SMC wing is really the brightest section of the stellar link between the SMC and the LMC. All of the individual blue stellar aggregates, including the region around the tip of the SMC wing appear to have been formed $\sim 10^{8}$ years ago, suggesting a common origin for these features. According to the tidal interaction model of Murai and Fujimoto (1980) the SMC came within $3 \mathrm{kpc}$ of the LMC some 200 million years ago. This approach may well have triggered the current burst of star formation that created the blue structures in the SMC wing and led to the birth of the blue stellar aggregates.

\section{The Magellanic Stream}

The Magellanic Stream is a narrow arc of neutral hydrogen extending roughly $180^{\circ}$ along a great circle from the NE periphery of the SMC, through the South Galactic Pole and onward to $1=90^{\circ}$, $\mathrm{b}=-30^{\circ}$ (Mathewson \& Ford, 1984). On the South Galactic Pole side the stream is composed of six large gas clouds, MSI-VI (Mathewson et al. 1977). HI surface densities range from a maximum of $5 \times 10^{20}$ atoms $\mathrm{cm}^{-2}$ within MSI to $3 \times 10^{19}$ atoms $\mathrm{cm}^{-2}$ for MSVI. We might expect to find an optical counterpart of these HI features if Magellanic Cloud stars were tidally torn out at the same time that the stream were formed (Murai \& Fujimoto 1980) or if the HI surface density in MSI-VI were high enough to initiate star formation. A complicating factor in any optical search is that the distance of the stream is not constrained directly from observations, and the distance, particularly near the tip, is unknown. The proximity of MSI to the Clouds and the smooth velocity gradient in this direction strongly suggest a $\sim 50 \mathrm{kpc}$ distance for this feature, whilst the dynamic models of Murai and Fujimoto imply a distance of $\sim 50 \mathrm{kpc}$ for the whole stream. 


\subsection{ABSORPTION STUDIES}

Songaila (1981) reported a positive detection of absorption by gas at $1^{h} 21.5,-50^{\circ} 04$ superimposed on the continuum of a background Seyfert galaxy. Unfortunately her limits on metallicity are not stringent enough to distinguish between primordial gas or Magellanic Cloud origin of the stream. Galaxy counts from UKST plates made by Mathewson et al. (1979) failed to reveal any significant absorption in the denser parts of the stream, whilst galaxy counts based on APM measures of UKST IIIaJ survey plates also fail to show any significant absorption $\left(A_{B}<0.2\right)$ within an area of over one hundred degrees ${ }^{2}$ centred on MSI. Fong et al. (1987) found a marginal indication of absorption in the Stream using galaxy counts from COSMOS measures of plates centred on the South Galactic Pole (SGP). In contrast, IRAS data revealed no significant $100 \mu \mathrm{m}$ flux, that could be attributed to dust in the Magellanic Stream near the SGP, and a further examination of the IRAS data in a region around MSI also failed to detect any $100 \mu \mathrm{m}$ flux, indicating that the Stream has a different dust content from that of the gas in the Galaxy. Clearly, given the relative insensitivity of galaxy counts for absorption measurements and the lack of any IRAS detection of dust in the Stream it would be worthwhile repeating Songaila's absorption studies using other bright AGNs as probes.

\subsection{OPTICAL EMISSION SURVEYS}

Mathewson et al. (1979) found no stellar component of the Stream from star counts on UKST IIIaJ survey plates, confirming the negative result from UBV photographic photometry of plates taken with the Uppsala Schmidt telescope. An earlier optical search for a faint blue stellar component by Philip (1976a,b) also produced a null result. In contrast, Sanduleak (1980) found a faint high latitude $\mathrm{CH}$-type carbon star at $23^{h} 46.7,+02^{\circ} 48$ coincident with the northern tip of the Magellanic Stream and estimated the distance to be $15 \mathrm{kpc}$. Richer and Westerlund (1983), in a survey of Carbon stars in Local Group Galaxies, reobserved Sanduleak's star and measured the radial velocity to be $-149 \mathrm{~km} \mathrm{~s}^{-1}, 150 \mathrm{~km} \mathrm{~s}^{-1}$ different from the Magellanic Stream velocity in this region. The carbon star was found to be spectroscopically similar to RGO 55 in $\omega$ Centauri, thereby placing it at a distance of $38 \mathrm{kpc}$.

Optical searches for stars of type A or earlier to $\mathrm{B}_{J}=18.5$ have been made by Recillas-Cruz (1982) near the tip of the stream, using UKST objective prism plates, whilst Tanaka and Hamajima (1982) used the Kiso Schmidt to conduct a two-colour UGR search to $R=17$ over 0.5 degrees $^{2}$ at $23^{h} 29.0,+08^{\circ} 44$. Both searches were again negative, suggesting that either the distance to the tip of the stream is much greater than $15 \mathrm{kpc}$ or that faint blue stars are not present in significant numbers.

Star counts to $\mathrm{B}_{J}=20.5$ on UKST IIIaJ plates over an area of 50 degrees $^{2}$ in the region of MSI were made by Brück and Hawkins (1983) using the COSMOS measuring machine. Apart from a marginal enhancement of faint images one degree east of the main HI concentration, no obvious stellar overdensity correlating with the HI contours was found. Since any excess stars were expected to consist of intermediate age objects, Brück and Hawkins (1983) consider that their negative result implies either that the age of Magellanic Stream is $>2 \times 10^{9}$ years or that the distance to the Stream is greater than that to the Clouds. We have repeated Brück and Hawkins' study using APM scans of UKST IIIaJ plates for fields 195,242,243,244 which cover the main area of MSI. This mini-survey of over 100 degrees $^{2}$ to a somewhat deeper limiting magnitude of $\mathrm{B}_{J}=22.5$ shows no apparent excess of stellar images. The marginal overdensity detected by Brück and Hawkins appears to be caused by a fluctuation in the background galaxy distribution. 
The problem with simply counting stars on photographic plates is that either the non-uniform response of the plate as a function of position or the Poisson noise from the image distribution can effectively swamp the true signal. This was certainly the reason that the reliable detection of an optical counterpart in the Magellanic Bridge region (Irwin et al. 1985, 1990a) awaited a multicolour survey. Consequently, a few years ago we initiated a deep UKST photographic $U, B_{J}, R$ search of 36 degrees $^{2}$ in the region around MSI (UKST field 195). The survey limits are $U=23, B_{J}=22$, and $R=21$. There is no apparent excess of a young blue stellar component, an intermediate-age red giant component, or of an older metal-poor giant population. Even if MSI were at several times the distance of the Clouds we would still have detected a stellar component.

In summary, all the direct optical searches for a stellar component of the Magellanic Stream have proven negative. It is possible that at least some of the gas in the system might have a nontidal origin. Mathewson and Schwarz (1976) pointed out that the gas in the Stream could be primordial in origin; whilst Mathewson, et al. (1977) proposed that the gas clouds in the Stream were formed in the turbulent wake of the Magellanic Clouds passing through a hot gaseous Galactic halo. The same notion of a hot gaseous Galactic halo was used by Meurer et al. (1985) who suggested that the inter-Cloud gas could have been preferentially stripped by gas drag.

\section{The Magellanic Plane}

Lynden-Bell (1976) pointed out that the Draco and Ursa Minor dwarf galaxies lie on the great circle defined by the Magellanic Stream, whilst the Sculptor dwarf lies close to MSII. Lynden-Bell suggested that these galaxies may be stellar remnants torn from a Greater Magellanic Galaxy in an ancient Magellanic Stream. At the same conference, Kunkel and Demers (1976) introduced the idea of a Magellanic Plane making an angle of $40^{\circ}$ with that defined by the Magellanic Stream. They demonstrated that most of the dwarf spheroidal satellites and remote globular clusters of the Milky Way lie within $7^{\circ}$ of this Plane. Indeed Kunkel (1979) suggested that all the dwarf spheroidals were associated with the Magellanic Clouds but were widely scattered due to the transverse spin. Within both scenarios there may also be a great tidal stream of faint stars around the sky which would be hard to detect against the stellar foreground of our Galaxy.

\subsection{THE SEXTANS DWARF SPHEROIDAL GALAXY}

A new Sculptor-type dwarf spheroidal galaxy at a distance of $85 \mathrm{kpc}$ was recently found by Irwin et al. (1990) in the constellation of Sextans at $10^{h} 10.5,-01^{\circ} 22$. The galaxy subtends a diameter of $1.5^{\circ}$, and has an ellipticity of 0.4 with a position angle for the major axis of $56^{\circ}$, measured from north through east. Preliminary colour-magnitude diagrams indicate that it has a very similar stellar mix to that found for Sculptor, whilst the absolute luminosity of Sextans is comparable to that of Ursa Minor and Draco. Given that both the APM and, independently, COMOS (MacGillivray et al. (1987), have surveyed most of the Southern sky for these types of objects it is interesting that the only additional dwarf spheroidal found so far lies within a few degress of the Magellanic Plane defined by Kunkel and Demers.

\subsection{FAINT HIGH GALACTIC LATITUDE CARBON STARS}

There are only a handful of high latitude carbon stars known at distances $>30 \mathrm{kps}$ (e.g. Margon $e t$ al. 1984, Mould et al. 1985 and references therein). One of these is Sanduleak's star near the tip of the Magellanic Stream mentioned in the previous section. With the exception of the carbon star found by Margon and co-workers, all of these carbon stars lie within $10^{\circ}$ of either the Magellanic 
Stream great circle or the Magellanic Plane of Kunkel and Demers. However, as the carbon stars were generally discovered serendipitously, have uncertain distances, and seem to be of several different types, it is hard to know what significance to attach to these observations with regard to possible Magellanic Cloud origin. In contrast, as part of a continuing systematic survey for very high redshift quasars (McMahon \& Irwin, 1989) we have found four high-latitude carbon stars with $\mathrm{R}$ magnitudes $\sim 16$ and $\mathrm{B}_{J}-\mathrm{R}$ colours $\sim 3.5$. Low-resolution spectra confirm the very red colour and show strong $\mathrm{CN}$ bands compared to the $\mathrm{C}_{2}$ strength (similar to the intermediate-age carbon stars found in Local Group galaxies), indicating that these stars are probably at distances $>50 \mathrm{kpc}$. The spectra, magnitudes and colours of the carbon stars are essentially identical, implying a similar distance for all of them. Furthermore, although we have surveyed a strip $100^{\circ}$ $x 6^{\circ}$ running along the celestial equator, all of the carbon stars lie within a $20^{\circ}$ substrip centred on $13^{h}, 0^{\circ}$ - coincident with the great circle defined by the Magellanic Stream. Star counts in the vicinity of the Carbon stars show nothing unusual (cf. Van den Bergh \& Lafontaine, 1984). We do not yet have accurate enough radial velocities to know whether these carbon stars are dynamically associated but given the ease with which they were detected and the general lack of similar candidates for the majority of the fields surveyed, it should be feasible to extend this survey to cover a significant fraction of both the northern and southern sky. Carbon stars may yet prove to be the only readily accessible optical manifestation of the possible tidal debris resulting from the disintegration of a Greater Magellanic Galaxy.

The author would like to thank the Royal Society and the organisers of IAU Symposium No. 148 for financial support to attend this meeting.

\section{References}

Albers, H., MacGillivray, H.T., Beard, S.M., Chromey, F.R. (1987), Astr. Astrophys. 182, L8.

Brück, M.T. (1975), M.N.R.A.S. 173, 327.

Brück, M.T. (1978), Astr. Astrophys. 68, 181.

Brück, M.T. (1980), Astr. Astrophys. 87, 92.

Brück, M.T., Hawkins, M.R.S. (1983) Astr. Astrophys. 124, 216.

Courtès, G., Viton, M., Sivan, J.P., Decher, R., Gary, A. (1984), Science 225, 179.

de Vaucouleurs, G. (1955), Astr. J. 60, 219.

de Vaucouleurs, G., Freeman, K. (1972), Vistas in Astronomy, 14, 163.

Fong, R., Jones, L.R., Shanks, T., Stevenson, P.R.F., Strong, A.W., Dawe, J.A., Murray, J.D. (1987), M.N.R.A.S. 224, 1059.

Freeman, K.C., Illingworth, G., Oemler, A. (1983), Astrophys. J. 272, 488.

Hatzidimitriou, D., Hawkins, M.R.S. (1989), M.N.R.A.S. 241, 667.

Hindman, J.V., Kerr, F.J., McGee, R.X. (1963), Aust. J. Phys. 16, 570.

Irwin, M.J., Kunkel, W.E., Demers, S. (1985), Nature 318, 160.

Irwin, M.J., Demers, S., Kunkel, W.E. (1990a), Astr. J. 99, 191.

Irwin, M.J., Bunclark, P.S., Bridgeland, M.T., McMahon, R.G. (1990b), M.N.R.A.S. 244, $16 \mathrm{P}$.

Johnson, P.G., Meaburn, J., Osman, A.M.I. (1982) M.N.R.A.S. 198, 985.

Kunkel, W.E. (1979), Astrophys. J. 228, 718.

Kunkel, W.E. (1980), IAU Symp 85, J.E. Hesser (ed.).

Kunkel, W.E., Demers, S. (1976), R. Greenwich Obs. Bull. 182, 241.

Lynden-Bell, D. (1976), M.N.R.A.S. 174, 695. 
Lynga, G., Westerlund, B.E. (1963) M.N.R.A.S. 127, 31.

MacGillivray, H.T., Bhatia, R.K., Beard, S.M., Dodd, R.J. (1987), ESO Workshop No. 27, M. Azzopardi, F. Matteucci (eds.) p.477.

Madore, B.F., Arp, H.C. (1979), Astrophys. J. Lett. 227, L103.

Marcelin, M. Boulesteix, J., Georgelin, Y. (1985), Nature 316, 705.

Margon, B., Aaronson, M., Liebert, J., Monet, D. (1984), Astr. J. 89, 274.

Mathewson, D.S., Ford, V.L. (1984), IAU Symp 108 Structure and evolution of the Magellanic Clouds, S. van den Bergh, K.S. de Boer (eds.) (Reidel: Dordrecht).

Mathewson, D.S., Schwarz, M.P. (1976), M.N.R.A.S. 176, 47P.

Mathewson, D.S., Schwarz, M.P., Murray, J.D. (1977), Astrophys. J. Lett. 217, L5.

Mathewson, D.S., Ford, V.L., Schwarz, M.P., Murray, J.D. (1979), IAU Symp 84 The Large Scale Characteristics of the Galaxy, W.B. Burton (ed.) p.547.

McGee, R.X., Haynes, R.F., Grognard, R.J.-M., Malin, D. (1986), M.N.R.A.S. 221, 543.

McMahon, R.G., Irwin, M.J. (1989), Gemini No.25, 6.

Meaburn, J. (1986), M.N.R.A.S. 223, 317.

Meurer, G.R., Bicknell, G.V., Gingold, R.A. (1985), Proc. Astron. Soc. Aust. 6, 195.

Mould, J.R., Schneider, D.P., Gordon, G.A., Aaronson, M., Liebert, J.W. (1985), Publ. astr. Soc. Pacific 97, 130.

Murai, T., Fujimoto, M. (1980), Publ. astr. Soc. Japan 32, 581.

Olszewski, W.E. (1988), IAU Symp 126, J.E. Grindlay, A.G. Philip (eds.) p.159.

Olszewski, W.E., Harris, H.C., Schommer, R.A., Canterna, R.W. (1988), Astr. J. $95,84$.

Philip, A.G.D. (1976a), Bull. A.A.S. 8352.

Philip, A.G.D (1976b), Bull. A.A.S. 8,532.

Recillas-Cruz, E. (1982), M.N.R.A.S. 201, 473.

Richer, H.B., Westerlund, B.E. (1983), Astrophys. J. 264, 114.

Sanduleak, N. (1969), Astr. J. 74, 877.

Sanduleak, N. (1980), Publ. astr. Soc. Pacific 92, 246.

Songaila, A. (1981), Astrophys. J. Lett. 243, L19.

Tanaka, K.I., Hamajima, K. (1982) Publ. astr. Soc. Japan 34, 417.

van den Bergh, S., Lafontaine, A. (1984), Publ. astr. Soc. Pacific 96, 869.

Wamsteker, W. (1981), Astron. Astrophys. Suppl. 43, 127.

Westerlund, B.E. (1989), Recent Developments of Magellanic Cloud Research, K.S. de Boer, F.

Spite, G. Stasinska (eds.) (Observatoire de Paris: Paris)p.159. 